\title{
EVALUACIÓN AGRONÓMICA DE SORGOS FORRAJEROS EN EL BAJÍO, MÉXICO
}

\author{
AGRONOMIC EVALUATION OF FORAGE \\ SORGHUMS AT THE BAJIO, MEXICO
}

\section{Jaime C. Rodríguez-Gómez ${ }^{1}$, Gabino García-De los Santos ${ }^{1}$, Adrián Hernández-Livera' y Miguel Hernández-Martínez ${ }^{2}$}

\begin{abstract}
'Colegio de Postgraduados, Programa en Recursos Genéticos y Productividad-Producción de Semillas, Montecillo, Texcoco, Estado de México, México. ${ }^{2}$ Instituto Nacional de Investigaciones Forestales, Agrícolas y Pecuarias, Campo Experimental Bajío, Celaya, Guanajuato, México.

*Autor de correspondencia (garciag@colpos.mx)
\end{abstract}

\section{RESUMEN}

El sorgo [Sorghum bicolor (L.) Moench] es un cultivo cada vez más utilizado como forraje en México, por lo que es importante contar con genotipos con atributos favorables. Se evaluaron 12 cultivares de sorgos forrajeros (SF), etanoleros (SE) y súper sorgos (SS) bajo condiciones de riego en el Campo Experimental Bajío del Instituto Nacional de Investigaciones Forestales Agrícolas y Pecuarias en Celaya Guanajuato, México en el ciclo agrícola primavera-verano de 2018, con el objetivo de determinar y comparar su potencial agronómico. El análisis de varianza mostró diferencias altamente significativas entre los tratamientos evaluados, ésto permitió diferenciar los genotipos en tardíos (SE), intermedios (SS) y precoces (SF) con base en sus días a floración, mientras que en altura de planta, los SS superaron a los demás grupos. En longitud de hoja los SS y SE fueron estadísticamente similares entre ellos y superiores a los SF. En cuanto a producción de biomasa verde los SS y SF presentaron un rendimiento de 93.24 y $81.55 \mathrm{t} \mathrm{ha}^{-1}$ respectivamente y fueron superiores a los $\mathrm{SE}$, los cuales obtuvieron en promedio $69.24 \mathrm{t} \mathrm{ha}^{-1}$. En el rendimiento de biomasa seca los SS fueron estadísticamente superiores a los demás grupos de sorgo con $20.89 \mathrm{t} \mathrm{ha}^{-1}$. En cuanto a la producción de azucares, aunque fue bajo, el mejor material fue SS-02 con $11.52^{\circ}$ Brix. Los súper sorgos superaron a los sorgos etanoleros en la mayoría de las variables evaluadas

Palabras clave: Sorghum bicolor L. Moench, Bajío, fenología, forraje.

\section{SUMMARY}

Sorghum [Sorghum bicolor (L.) Moench] is a crop increasingly used as forage in Mexico; thus, it is important to have genotypes with favorable attributes. Twelve cultivars of forage sorghum (FS), ethanol (ES) and super sorghum (SS) were evaluated under irrigated conditions at the Bajio Experiment Station of the National Institute of Forestry, Agriculture and Livestock Research in Celaya Guanajuato, Mexico in the Spring-Summer agricultural cycle of 2018, with the aim of determining and comparing their agronomic potential. The analysis of variance showed highly significant differences between the evaluated treatments, which allowed differentiating the genotypes into late (ES), intermediate (SS) and early (FS) based on days to flowering, while in plant height the SS outperformed the other groups. In leaf length, SS and ES were statistically similar between them, and superior to FS. Regarding green biomass production, SS and FS produced a yield of 93.24 and $81.55 \mathrm{t} \mathrm{ha}^{-1}$ respectively and were higher than ES, which produced an average of $69.24 \mathrm{t}$ $\mathrm{ha}^{-1}$. In dry biomass yield the SS were statistically superior to the other groups of sorghum with $20.89 \mathrm{tha}^{-1}$. Regarding sugar production, although it was low, the best material was SS- 02 with $11.52^{\circ}$ Brix. Super sorghums outperformed ethanol sorghums in most of the variables evaluated.

Index words: Sorghum bicolor L. Moench, Bajio, forage, phenology.

\section{INTRODUCCIÓN}

El sorgo [Sorghum bicolor (L.) Moench] es un cultivo cada vez más utilizado como forraje en México, en el ciclo agrícola 2020 se sembraron 151,540 hectáreas de sorgo forrajero con rendimientos promedio de 23.7 t ha ${ }^{-1}$ (SIAP, 2021), ésto se debe a su tolerancia a sequía, especialmente en etapa vegetativa (Castro et al., 2000), en comparación con el maíz, debido que produce mayor cantidad de materia seca por unidad de agua; una planta de sorgo consume de 80 a $100 \mathrm{~mL}$ de agua menos que el maíz en etapa reproductiva (Ajeigbe et al., 2018).

Las hojas del sorgo tienen una cubierta cerosa y por regulación osmótica evita la deshidratación; además, aumenta la absorción de agua a través de su sistema radical fibroso, denso y ramificado, que permite tener una mayor exploración del suelo y absorción de nitrógeno (Legarto, 2000); así mismo, el sorgo evita la pérdida transpiracional de agua a través del hábito de crecimiento vertical de las hojas, tolera la salinidad y el manejo intensivo de corte (Dar et al., 2018), en comparación con otros cultivos forrajeros como maíz, alfalfa, avena, etc; sin embargo, en suelo fértil y con disponibilidad de riego, el sorgo no compite en rendimiento de materia seca con maíz o alfalfa. En los últimos años, la falta de agua para riego y las sequias recurrentes han restringido el cultivo de maíz y alfalfa en la región del Bajío; por tal razón, es importante la evaluación de nuevos cultivares de sorgo con características agronómicas sobresalientes que permitan 
mejores rendimientos y mayor valor nutricional para ser utilizados en la alimentación humana y animal.

En México, el $27.7 \%$ de la superficie se cultiva en la modalidad riego, de la cual el $53 \%$ de la superficie se cultiva en el ciclo primavera-verano y el resto en el otoñoinvierno. Con respecto a la productividad del cultivo de sorgo, el rendimiento de grano promedio nacional en riego en 2020 fue de $5.20 \mathrm{t} \mathrm{ha}^{-1} \mathrm{y}$ en temporal en $2.47 \mathrm{t} \mathrm{ha}^{-1}$ (SIAP, 2021). La región de El Bajío aporta aproximadamente el $16 \%$ de la producción nacional de sorgo. En Guanajuato, la producción se concentra en ocho de los 46 municipios del estado, los que aportan el $78 \%$ de la producción. Estos municipios se localizan en el sureste del estado (SIAP, 2021). En este cotexto, el objetivo del presente estudio fue determinar y comparar el potencial de producción de biomasa de 12 cultivares de sorgo forrajero, etanolero y súper sorgo.

\section{MATERIALES Y MÉTODOS}

El estudio se llevó a cabo en el Campo Experimental Bajío del Instituto Nacional de Investigaciones Forestales, Agrícolas y Pecuarias (INIFAP), situado a $20^{\circ} 32^{\prime} 05^{\prime \prime}$ latitud norte y $100^{\circ} 48^{\prime} 49^{\prime \prime}$ longitud oeste, a $1750 \mathrm{msnm}$, con un clima semicálido y temperaturas que van de 18 a $22^{\circ} \mathrm{C}$, la precipitación pluvial varía de 600 hasta $1000 \mathrm{~mm}$ anuales, presentando un promedio anual de $670.3 \mathrm{~mm}$, suelo de tipo vertisol (SPP, 1980).

Se evaluaron 12 cultivares de sorgo, tres forrajeros: Silo Máster, Silo Miel, Silage King (testigo), seis etanoleros: ET-V1, ETV-2, ET-V3, ET-V4, ET-V5 y RB-Cañero (testigo), y tres súper sorgos: SS-02, SS-09 y SS-35.

Se empleó un diseño experimental de bloques completos al azar con cuatro repeticiones, la unidad experimental fue de cuatro surcos de cinco metros de longitud y una distancia entre surcos de $0.76 \mathrm{~m}$, la parcela útil fue de un surco central, la siembra se realizó manualmente con depósito continuo de semilla (chorrillo) a razón de $40 \mathrm{~g}$ por cada unidad experimental (11.29 kg ha-1).

Al momento de la siembra se aplicó la fórmula de fertilización 90-40-00 ( $\mathrm{kg}$ ha-1 de $\mathrm{N}-\mathrm{P}_{2} \mathrm{O}_{5}-\mathrm{K}_{2} \mathrm{O}$ ); posteriormente, se aplicaron otros $90 \mathrm{~kg} \mathrm{ha}^{-1}$ de nitrógeno al momento de la primera escarda, 35 días después de siembra. Se programaron cuatro riegos con calendario 0-35-75-110 días después de la siembra; sin embargo, dado que la precipitación durante el ciclo de cultivo fue por arriba de lo normal (750 vs 520 mm), sólo se dio el riego de nacencia. Para el control de maleza se aplicó en premergencia el herbicida Gesaprim Combi (Atrazina + Terbutrina) $2.0 \mathrm{~L} \mathrm{ha}^{-1}$. A los 45 días después de la siembra se presentó la plaga de pulgón amarillo del sorgo (Melanaphis sacchari), la cual se controló con el insecticida Toretto (Sulfoxaflor) en dosis de $50 \mathrm{~mL} \mathrm{ha}^{-1}$.

Durante el desarrollo del cultivo se registraron las variables: 1) días a floración (DF), 2) altura total de la planta (AP) en $m, 3$ ) altura a la base de la panoja (ABP) en $m, 4$ ) longitud de la panoja (LP) en $\mathrm{m}, 5$ ) altura a la hoja bandera (AHB) en $\mathrm{m}, 6$ ) número de hojas (NH), 7) longitud de hoja $(\mathrm{LH})$ en $\mathrm{cm}, 8)$ ancho de hoja $(\mathrm{AH})$ en $\mathrm{cm}, 9)$ número de nudos, 10 ) sólidos solubles ('Brix) para lo cual se realizó un corte transversal en el séptimo nudo en dirección apical-basal de la planta y se tomaron muestras del jugo sobre las cuales se hicieron las determinaciones mediante un refractómetro (GrandBeing, Modelo 090, Grandbeing Technology, Orlando, Florida, EUA), 11) rendimiento de biomasa verde (RBV) en tha-1 y 12) rendimiento de biomasa seca (RBS) con secado en invernadero de la biomasa verde; para determinar el porcentaje de materia seca se utilizó la siguiente ecuación: \% MS ( $\frac{\text { rendimiento de biomasa seca }}{\text { rendimiento de biomasa verde }}$ ) $\times 100$ en $\mathrm{tha}^{-1}$.

El modelo lineal aditivo utilizado fue el correspondiente al diseño de bloques completos al azar. Para cada una de las variables se corrieron los análisis de varianza empleando el paquete estadístico SAS (SAS Institute, 2011) y se utilizó la prueba de Tukey $(P \leq 0.05)$ para la comparación de medias entre tratamientos, la prueba de Scheffé para realizar contrastes entre los grupos de sorgos forrajeros, súper-sorgos y etanoleros.

\section{RESULTADOS Y DISCUSIÓN}

El análisis de varianza mostró diferencias significativas $(P \leq 0,05)$ entre los cultivares de sorgo para todas las variables evaluadas, mientras que entre bloques sólo hubo diferencias significativas en las variables altura total de planta y altura a la base de la panoja, lo que puede atribuirse a las diferencias del terreno o al manejo agronómico del experimento ya que el coeficiente de variación fue bajo (Cuadro 1).

\section{Comparaciones de medias}

Con base en los días a floración (Cuadro 2) se pudieron identificar tres grupos de genotipos, cultivares tardíos como: ET-V1 (89 días), ET-V5 (88 días) y RB Cañero (88 días), intermedios: ET-V4 (85 días), ET-V2 (82 días, ET-V3 (82 días), SS 08 (78 días), SS 09 (76 días), S. Máster (75 días) y SS 35 (74 días); los precoces fueron Silage King (71 días) y Silo Miel (72 días). La duración de las etapas fenológicas del sorgo está determinada por genotipo y ambiente, dentro del cual la temperatura y precipitación muestran mayor influencia y pueden atrasar o alargar el 
Cuadro 1. Cuadrados medios y significancia estadística de las variables estudiadas.

\begin{tabular}{|c|c|c|c|c|}
\hline \multirow{2}{*}{ Variables } & \multicolumn{2}{|c|}{ Fuentes de variación } & \multirow{2}{*}{ CV (\%) } & \multirow{2}{*}{$R^{2}$} \\
\hline & Cultivares de sorgo & Bloques & & \\
\hline Días a floración & $170.65 * *$ & 0.39 & 0.96 & 0.99 \\
\hline Altura total de planta & $1.41 * *$ & $0.13 *$ & 5.77 & 0.92 \\
\hline Altura a la base de la panoja & $1.09 * *$ & $0.09 *$ & 5.11 & 0.93 \\
\hline Longitud de la panoja & $0.06 * *$ & 0.01 & 30.95 & 0.63 \\
\hline Altura a la hoja bandera & $0.98 * *$ & 0.07 & 4.91 & 0.93 \\
\hline Rendimiento de biomasa verde & $177.27 \star \star$ & 40.56 & 19.71 & 0.66 \\
\hline Rendimiento de biomasa seca & $11.19 * \star$ & 1.56 & 25.64 & 0.59 \\
\hline Número de hojas & $12.57 * \star$ & 0.53 & 5.33 & 0.89 \\
\hline Longitud de hoja & $288.08 * \star$ & 14.62 & 4.99 & 0.74 \\
\hline Ancho de hoja & $4.26 * *$ & 1.35 & 10.14 & 0.57 \\
\hline Número de nudos & $12.08 * *$ & 0.80 & 6.43 & 0.83 \\
\hline Grados Brix & $12.98 * \star$ & 2.72 & 12.21 & 0.63 \\
\hline
\end{tabular}

* Significativo $(P \leq 0.05)$, ** Altamente significativo $(P \leq 0.01)$.

ciclo de vida de las plantas (Solórzano, 2007). Al respecto, Soto y Hernández (2012) observaron una fluctuación en la etapa de emergencia a floración de 56 a 71 días después de siembra y de floración a madurez de 35 a 37 días, mientras que González et al. (2005) reportaron de 57 a 69 días después de siembra para inicio de floración en nueve cultivares de sorgo forrajero, lo mismo que reportaron Kapanigouda et al. (2013) al evaluar 48 líneas de sorgo. En este estudio Súper Sorgo 09 presentó la mayor altura de planta $(3.43 \mathrm{~m})$ y fue superior a los reportados en otros estudios con otros genotipos. Amador y Boschini (2000) reportaron una altura máxima de $2.74 \mathrm{~m}$, mientras que Afzal et al. (2012) observaron una altura de planta de 1.94 $m$ en sorgos forrajeros; es posible que las diferencias se deban a que en la actualidad existen genotipos nuevos con mejores características y mayor potencial que los evaluados anteriormente. En rendimiento de biomasa verde, el cultivar más sobresaliente fue Súper Sorgo 02 (119.7 t ha $\left.^{-1}\right)$; el resto de los cultivares no mostraron diferencias significativas con respecto a la variable en cuestión. Para la variable rendimiento de biomasa seca, Súper Sorgo 02 (28.4 t ha-1) fue el cultivar con mayor producción, superando a la mayoría de los sorgos dulces ( 18 a 32 ton ha $^{-1}$ ) reportados por Wu et al. (2010). Para contenido de sólidos solubles ( ${ }^{\circ} \mathrm{Brix}$ ), el genotipo que presentó mayor cantidad de sólidos solubles fue Súper Sorgo 02 (11.52 ${ }^{\circ}$ Brix) y el de menor contenido fue RB Cañero (8.18 ${ }^{\circ}$ Brix). Estos resultados difieren de los reportados por Almodares et al. (2006), quienes encontraron que los sólidos solubles acumulados en el tallo de sorgo dulce varían de 14 a 23 'Brix; la diferencia en el contenido de sólidos solubles encontrados en este estudio pudiera atribuirse a que la evaluación se realizó durante la época de floración y no durante la etapa de madurez del grano, que es donde se acumula la mayor cantidad de azúcares (Ferrari y CharlesEdwards, 1986).

En cuanto a los diferentes grupos de sorgos (forrajero, etanoleros y súper sorgos), para la variable altura total de planta hubo significancia estadística entre grupos, donde la mayor altura fue para los SS (3.12 m), lo que influye en mayor rendimiento en biomasa verde y seca; la menor altura la presentaron los SF $(2.38 \mathrm{~m})$. Para el rendimiento de biomasa verde destaca que los SS y SF fueron estadísticamente iguales con 93.24 y 81.55 $\mathrm{t}$ ha $^{-1}$ respectivamente, pero ambos fueron superiores a los SE, los cuales obtuvieron en promedio $69.24 \mathrm{t}$ ha $^{-1}$. Para la variable rendimiento de biomasa seca, estadísticamente mostraron superioridad los SS con 20.89 t ha $^{-1}$, en comparación con los SF y SE, 16.97 y 16.21 t ha ${ }^{-1}$, respectivamente. En cuanto a contenido de sólidos solubles, no hubo diferencias estadísticas entre los grupos evaluados.

\section{Contrastes}

Los diferentes tipos de sorgos se agruparon en Súper sorgos (SS), Sorgos etanoleros (SE) y Sorgos forrajeros (SF), cada grupo constó de tres cultivares para realizar contrastes en comparaciones balanceadas mediante la prueba de Scheffé.

En el Cuadro 3 se muestra la comparación de medias entre los tres grupos de sorgo para las variables evaluadas. 
En lo que respecta a la variable días a floración, los tres grupos fueron estadísticamente diferentes, siendo más tardíos los SE (87 días) y más precoces los SF (73 días).

En conclusión se observó que los súper sorgos superaron a los sorgos etanoleros en la mayoría de las variables evaluadas, seguido por los sorgos forrajeros, destacando la producción de biomasa verde y biomasa seca, cabe resaltar que los ${ }^{\circ}$ Brix de los cultivares de sorgo evaluados fueron muy bajos en comparación con los reportados en otros estudios, los mejores cultivares dentro de cada grupo fueron Súper Sorgo 02, Silage King y ET-V1, para Super Sorgo, Sorgo Forrajero y Sorgo Etanolero, respectivamente.

\section{BIBLIOGRAFÍA}

Afzal M., A. Ahmad and A. U. H. Ahmad (2012) Effect of nitrogen on growth and yield of sorghum forage (Sorghum bicolor (L.) Moench $\mathrm{CV}$ ) under three cuttings system. Agronomical Research in Moldavia 45:57-64

Ajeigbe H. A., F. M. Akinseye, K. Ayuba and J. Jonah (2018) Productivity and water use efficiency of sorghum [(Sorghum bicolor (L.) Moench.] grown under different nitrogen applications in Sudan savanna zone, Nigeria. International Journal of Agronomy
2018:7676058, https://doi.org/10.1155/2018/7676058

Almodares A., M. R. Hadi and H. Ahmadpour (2008) Sorghum stem yield and soluble cabohydrates under different salinity levels. African Journal of Biotechnology 22:4051-4055.

Amador A. L. y C. Boschini (2000) Calidad nutricional de la planta de sorgo negro forrajero (Sorghum alum) para alimentación animal. Agronomía Mesoamericana 11:79-84.

Castro N. S., J. Ortiz C., M. C. Mendoza C. y F. Zavala G. (2000) Producción de biomasa en líneas de sorgo como respuesta al estrés hídrico. Revista Fitotecnia Mexicana 23:321-334.

Dar R. A., E. A. Dar, A. Kaur and U. G. Phutela (2018) Sweet sorghum -a promising alternative feedstock for biofuel production Renewable and Sustainable Energy Reviews 82:4070-4090, https://doi.org/10.1016/j.rser.2017.10.066

Ferrari R. and D. A. Charles-Edwards (1986) A comparative analysis of the growth of sweet and forage sorghum crops. I. Dry matter production, phenology, and morphology. Australian Journal of Agricultural Research 37:495-512, https://doi.org/10.1071/ AR9860495

González T. R. O., W. Y. Sequera M. e Y. Graterol (2005) Comportamiento de nueve cultivares de sorgo forrajero en Portuguesa, Venezuela. Pastos 35:151-162.

Kapanigowda M. H., R. Perumal, R. M. Aiken, T. J. Herald, S. R. Bean and C. R. Little (2013) Analyses of sorghum (Sorghum bicolor (L.) Moench) lines and hybrids in response to early-season planting and cool conditions. Canadian Journal of Plant Science 93:773784, https://doi.org/10.4141/cjps2012-311

Legarto J. (2000) L'utilisation en ensilage plante entière des sorghos grains et sucriers: intérêts et limites pour les régions sèches. Fourrages 163:323-338.

Cuadro 2. Comparación de medias entre cultivares de sorgo.

\begin{tabular}{|c|c|c|c|c|c|c|c|c|c|c|c|c|}
\hline Variables & ET-V1 & ET-V2 & ET-V3 & ET-V4 & ET-V5 & SS 02 & SS 09 & SS 35 & $\begin{array}{c}\text { S. } \\
\text { Máster }\end{array}$ & S. Miel & S. King & $\begin{array}{c}\text { RB. } \\
\text { Cañero }\end{array}$ \\
\hline DF & 89 a & $82 \mathrm{c}$ & $82 \mathrm{c}$ & $85 b$ & $88 a$ & $78 d$ & $76 \mathrm{e}$ & $74 \mathrm{e}$ & $75 e$ & $72 f$ & $71 \mathrm{f}$ & 88 a \\
\hline $\operatorname{ATP}(\mathrm{m})$ & $2.30 \mathrm{~d}$ & $2.10 \mathrm{e}$ & $2.30 \mathrm{~d}$ & $2.89 b$ & $2.16 \mathrm{e}$ & $3.16 b$ & $3.43 \mathrm{a}$ & $2.75 c$ & $2.70 \mathrm{~d}$ & $2.26 \mathrm{e}$ & $2.20 \mathrm{e}$ & $2.50 \mathrm{~d}$ \\
\hline RBV $\left(\mathrm{t} \mathrm{ha}^{-1}\right)$ & $73.3 b$ & $54.9 \mathrm{~b}$ & $58.5 b$ & $69.4 \mathrm{~b}$ & $64.9 \mathrm{~b}$ & $119.7 \mathrm{a}$ & $75.7 \mathrm{~b}$ & $84.3 b$ & 79.6 b & $76.6 \mathrm{~b}$ & $88.4 b$ & $58.3 b$ \\
\hline RBS (t ha-1) & $18.60 \mathrm{~b}$ & $12.00 \mathrm{~b}$ & $15.00 \mathrm{~b}$ & $16.00 \mathrm{~b}$ & $13.90 \mathrm{~b}$ & $28.40 \mathrm{a}$ & $18.70 \mathrm{~b}$ & $15.60 \mathrm{~b}$ & $17.40 \mathrm{~b}$ & $15.40 \mathrm{~b}$ & $17.90 \mathrm{~b}$ & $11.10 \mathrm{~b}$ \\
\hline RMS $\left(\mathrm{t} \mathrm{ha}^{-1}\right)$ & $67.1 \mathrm{a}$ & $58 \mathrm{~b}$ & $67.6 \mathrm{a}$ & $59.2 \mathrm{~b}$ & $56.8 b$ & $61.8 \mathrm{~b}$ & $65.1 \mathrm{~b}$ & $48.5 \mathrm{c}$ & $57.3 \mathrm{~b}$ & $53.2 b$ & $53.4 b$ & $49.2 \mathrm{~b}$ \\
\hline${ }^{\circ}$ Brix & $9 b$ & $10.6 b$ & $11.1 \mathrm{~b}$ & $11.2 \mathrm{~b}$ & $8.65 b$ & $11.52 \mathrm{a}$ & $8.81 \mathrm{~b}$ & $8.31 \mathrm{~b}$ & $9.43 b$ & $8.50 \mathrm{~b}$ & $8.31 \mathrm{~b}$ & $8.18 \mathrm{c}$ \\
\hline
\end{tabular}

Medias con la misma letra en las columnas son estadísticamente iguales (Tukey, $\mathrm{P} \leq 0.05)$. DF: días a floración, AP : altura total de planta, RBV: rendimiento de biomasa verde, RBS: rendimiento de biomasa seca.

Cuadro 3. Comparación de medias de las variables de cada grupo de sorgos: sorgos forrajeros, súper sorgos y etanoleros.

\begin{tabular}{lccc}
\hline Variable & Sorgos forrajeros & Súper sorgos & Sorgos etanoleros \\
\hline Días a floración & $73.00 \mathrm{c}$ & $76.00 \mathrm{~b}$ & $87.00 \mathrm{a}$ \\
Altura total de planta $(\mathrm{m})$ & $2.38 \mathrm{c}$ & $3.12 \mathrm{a}$ & $2.46 \mathrm{~b}$ \\
Rendimiento de biomasa verde $\left(\mathrm{t} \mathrm{ha}^{-1}\right)$ & $81.55 \mathrm{ab}$ & $93.24 \mathrm{a}$ & $69.24 \mathrm{c}$ \\
Rendimiento de biomasa seca $\left(\mathrm{t} \mathrm{ha}^{-1}\right)$ & $16.97 \mathrm{~b}$ & $20.89 \mathrm{a}$ & $16.21 \mathrm{~b}$ \\
Grados brix & $8.75 \mathrm{a}$ & $9.55 \mathrm{a}$ & $9.63 \mathrm{a}$ \\
\hline
\end{tabular}

Medias con la misma letra en las columnas son estadísticamente iguales (Scheffé, $\mathrm{P} \leq 0.05)$. 
SAS Institute (2011) SAS/STAT ${ }^{\oplus} 9.3$ User's Guide. SAS Institute Inc., Cary, North Carolina, USA. 8621 p.

SIAP, Servicio de Información Agroalimentaria y Pesquera (2021) Anuario estadístico de la producción agrícola. Secretría de Agricultura y Desarrollo Rural. Ciudad de México. https://nube.siap.gob.mx/ cierreagricola/ (Octubre 2021).

Solórzano V. E. (2007) Guías Fenológicas para Cultivos Básicos. Editorial Trillas. México, D. F. 152 p

Soto C. F. y N. Hernández C. (2012) Influencia de tres fechas de siembra en el crecimiento y rendimiento de especies de cereales cultivadas en condiciones tropicales. Parte II. Cultivo del sorgo (Sorghum bicolor L. Moench var. Isiap Dorado). Cultivos Tropicales 33:50 54.

SPP, Secretaría de Programación y Presupuesto (1980) Sintesis geográfica de Guanajuato. Secretaría de Programación y Presupuesto. México, D. F. 26 p.

Wu X., S. Staggenborg, J. L. Propheter, W. L. Rooney, J. Yu and D. Wang (2010) Features of sweet sorghum juice and their performance in ethanol fermentation. Industrial Crops and Products 31:164170, https://doi.org/10.1016/j.indcrop.2009.10.006 
